ARTICLE

Received 11 Feb 2016 | Accepted 6 May 2016 | Published 14 Jun 2016

DOI: $10.1038 /$ ncomms11856

OPEN

\title{
A luciferin analogue generating near-infrared bioluminescence achieves highly sensitive deep-tissue imaging
}

Takahiro Kuchimaru ${ }^{1, \star}$, Satoshi Iwano ${ }^{2, \star}$, Masahiro Kiyama ${ }^{2}$, Shun Mitsumata1, Tetsuya Kadonosono ${ }^{1}$ Haruki Niwa ${ }^{2}$, Shojiro Maki ${ }^{2} \&$ Shinae Kizaka-Kondoh ${ }^{1}$

In preclinical cancer research, bioluminescence imaging with firefly luciferase and D-luciferin has become a standard to monitor biological processes both in vitro and in vivo. However, the emission maximum $\left(\lambda_{\max }\right.$ ) of bioluminescence produced by D-luciferin is $562 \mathrm{~nm}$ where light is not highly penetrable in biological tissues. This emphasizes a need for developing a red-shifted bioluminescence imaging system to improve detection sensitivity of targets in deep tissue. Here we characterize the bioluminescent properties of the newly synthesized luciferin analogue, AkaLumine- $\mathrm{HCl}$. The bioluminescence produced by $\mathrm{AkaLumine}-\mathrm{HCl}$ in reactions with native firefly luciferase is in the near-infrared wavelength ranges $\left(\lambda_{\max }=677 \mathrm{~nm}\right)$, and yields significantly increased target-detection sensitivity from deep tissues with maximal signals attained at very low concentrations, as compared with D-luciferin and emerging synthetic luciferin CycLuc1. These characteristics offer a more sensitive and accurate method for non-invasive bioluminescence imaging with native firefly luciferase in various animal models.

\footnotetext{
${ }^{1}$ School of Life Science and Technology, Tokyo Institute of Technology, 4259, Nagatsuta, Midori-ku, Yokohama 226-8501, Japan. ${ }^{2}$ Graduate School of Informatics and Engineering, The University of Electro-Communications, 1-5-1 Chofugaoka, Chofu, Tokyo 182-8585, Japan. * These authors contributed equally to this work. Correspondence and requests for materials should be addressed to S.K.-K. (email: skondoh@bio.titech.ac.jp).
} 
B ioluminescence is generated through an oxidation reaction between the enzyme luciferase and its substrate, luciferin ${ }^{1,2}$. In preclinical cancer research, bioluminescence imaging (BLI) with firefly luciferase (Fluc) and D-luciferin has become a standard to monitor tumour growth, protein-protein interactions and specific molecular activity in cell lines and mouse models ${ }^{3-6}$. The emission maximum $\left(\lambda_{\max }\right)$ of bioluminescence produced by D-luciferin is $562 \mathrm{~nm}$. In the visible light spectrum, haemoglobin $(\lambda=\sim 415-577 \mathrm{~nm})$ and melanin $(\lambda<600 \mathrm{~nm})$ dominate absorption, hindering the detection of signals emanating from deep tissues ${ }^{7,8}$. In recent times, cyclic alkylaminoluciferin (CycLuc1) $\left(\lambda_{\max }=604 \mathrm{~nm}\right)$ demonstrated improved detection of targets in vivo by a significantly increased bioluminescence production over D-luciferin ${ }^{9}$. However, its short $\lambda_{\text {max }}$ keep it from maximizing the detection sensitivity of targets in deep tissue.

To address this issue, efforts have been made to develop a BLI system that uses near-infrared (NIR) wavelength range of around $650-900 \mathrm{~nm}$, where signal penetration through biological tissues is greatly improved ${ }^{7}$. NIR bioluminescence for in vivo BLI has been achieved with a synthetic D-luciferin analogue $\left(\lambda_{\max }=706 \mathrm{~nm}\right)^{10}$ and bioluminescence resonance energy transfer in aminoluciferin-conjugated NIR fluorescence dyes $\left(\lambda_{\max }<800 \mathrm{~nm}\right)^{11}$. However, these attempts have failed to show a significant improvement in the detection of targets in vivo when compared with D-luciferin, owing to their low bioluminescence production.

We previously synthesized a novel luciferin analogue, AkaLumine, by replacing the aromatic structure in D-luciferin with a benzothiazole moiety ${ }^{12}$. Notably, the $\lambda_{\max }$ of bioluminescence produced by AkaLumine is $675 \mathrm{~nm}$, which displays high penetration in vivo. However, the high hydrophobicity of AkaLumine causes poor water solubility
$(<2 \mathrm{mM})$, which is a major drawback for its use in vivo. We thus screened water-soluble derivatives of AkaLumine. Here we report one of the derivatives, AkaLumine hydrochloride (AkaLumine- $\mathrm{HCl}$ ) that is applicable for in vivo BLI. Akalumine$\mathrm{HCl}$ emits light in the NIR wavelength ranges $\left(\lambda_{\max }=677 \mathrm{~nm}\right)$ in reactions with native Fluc and yields increased detection sensitivity from deep-tissue targets with maximal signals attained at very low concentrations, as compared with D-luciferin and CycLuc1. These characteristics offer a more sensitive and accurate method for non-invasive BLI in various animal models using native Fluc.

\section{Results}

Characterization of AkaLumine-HCl. AkaLumine was able to detect targets in deep tissues of mouse tumour models with a significantly higher sensitivity than D-luciferin at the same concentration (Supplementary Fig. 1). To improve applicability of AkaLumine for in vivo imaging, we synthesized AkaLumine- $\mathrm{HCl}$ (Fig. 1a), which has high solubility in water $(<40 \mathrm{mM})$. AkaLumine-HCl emitted NIR-shifted bioluminescence $\left(\lambda_{\max }=677 \mathrm{~nm}\right)$ in reactions with Fluc (Fig. 1b) and greatly improved tissue penetration efficiency (Fig. 1c): AkaLumine- $\mathrm{HCl}$ bioluminescence showed 5- and 8.3-fold higher penetration than D-luciferin bioluminescence, and 3.7- and 6.7-fold higher penetration than CycLuc1 bioluminescence in 4 - or 8 -mm-thick tissue sections, respectively (Fig. 1c). A 60-fold higher concentration of D-luciferin was required to obtain signal intensity comparable to AkaLumine- $\mathrm{HCl} \quad(150 \mu \mathrm{M}$ versus $2.5 \mu \mathrm{M}$, respectively; Supplementary Fig. 2). These results suggest that AkaLumine$\mathrm{HCl}$ is better suited for the detection of targets in deep tissue. a<smiles>CN(C)c1ccc(/C=C/C=C/C2=NC(C(=O)O)CC2)cc1</smiles><smiles>O=C(O)C1CSC(c2nc3ccc(O)cc3s2)=N1</smiles>

D-luciferin<smiles>O=C(O)C1CSC(c2nc3cc4c(cc3s2)NCC4Cl)=N1</smiles>

C

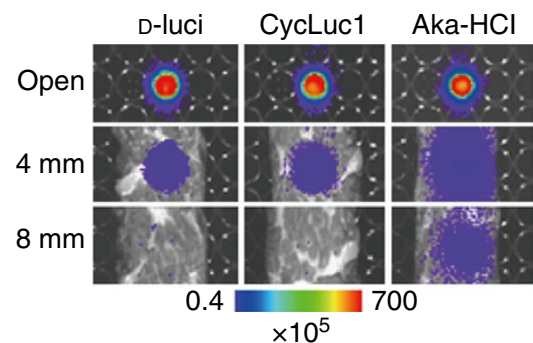

b

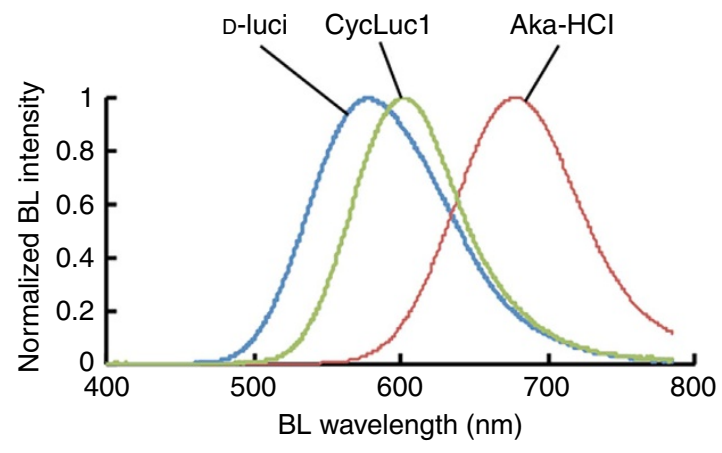

D-luci CycLuc1
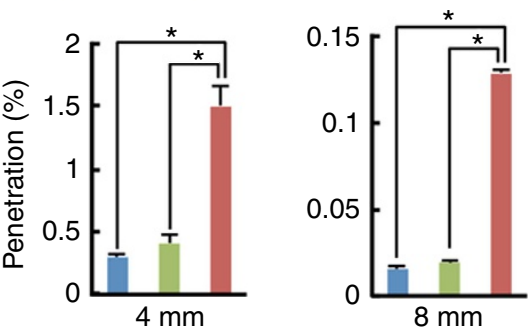

Figure 1 | Properties of AkaLumine-HCl. (a) Chemical structures of AkaLumine-HCl, D-luciferin and CycLuc1. (b) Bioluminescence emission spectra of D-luciferin (D-luci), CycLuc1 or AkaLumine-HCl (Aka-HCl). (c) Biological tissue penetration efficiency of bioluminescence generated by D-luci, CycLuc1 or $\mathrm{Aka}-\mathrm{HCl}$. Penetration efficiency indicates the relative bioluminescence intensities of the wells covered with a biological tissue (4- or 8-mm thick) versus open wells (open). $n=3,{ }^{\star} P<0.05$ (t-test). Error bars indicate s.e.m. 
To evaluate the properties of AkaLumine- $\mathrm{HCl}$ in BLI at the cellular level, bioluminescence signals from LLC/luc (mouse lung carcinoma) and MDA-MB-231/luc (human breast carcinoma) cancer cell lines with constitutive expression of Fluc were analysed after treatments with increasing concentrations of D-luciferin, CycLucl and AkaLumine-HCl (Fig. 2). Interestingly, in both LLC/ luc and MDA-MB-231/luc cells treated with AkaLumine- $\mathrm{HCl}$, the signal was maximal at lower concentrations (by $2.5 \mu \mathrm{M}$ ), whereas the bioluminescence generated by D-luciferin and CycLucl increased as concentrations increased further, not appearing to reach a maximal signal even at $250 \mu \mathrm{M}$ (Fig. 2). The bioluminescent signals in the NIR range, which provide a better correlation with in vivo images, from AkaLumine- $\mathrm{HCl}$ were strong and less influenced by their concentrations, whereas the ones from CycLuc1 and D-luciferin showed a dose-dependent increase and were weak at lower concentrations that are commonly reached in vivo by ordinary mouse experiments (Fig. 2b,d). These results illustrate the differences in dose dependence among the three agents, with AkaLumine- $\mathrm{HCl}$ appearing to be optimally effective at much lower doses than the others, an effect particularly evident when imaging in the NIR range. Both AkaLumine- $\mathrm{HCl}$ and CycLucl showed less dose dependency than D-luciferin and decreased in bioluminescence production at a high concentration in the reaction with recombinant Fluc protein (Supplementary Fig. 3) probably due to their low $K_{\mathrm{m}}$ values (Table 1), which lead to saturation of Fluc with them at relatively low concentrations, and product inhibition effects, in which the mechanism is reported for D-luciferin ${ }^{13,14}$ but still unknown for CycLucl and AkaLumine-HCl. However, CycLuc1 showed a clear dose-dependent bioluminescence production with Fluc expressed in the cells (Fig. 2) at the same concentration range as the reaction with recombinant Fluc protein (Supplementary Fig. 3). This dose dependency of CycLucl is probably due to limited cell-membrane permeability, which is a major factor limiting enzyme-substrate interactions in cells, influencing bioluminescence production rate ${ }^{15-17}$. Comparison of bioluminescence production levels in intact and lysed cells shortly after treatments with the substrates revealed the differences in cell-membrane permeability among them: the cell-membrane permeability of AkaLumine- $\mathrm{HCl}$ was much greater than the others (Fig. 3a). Furthermore, pretreatment of the cells with a
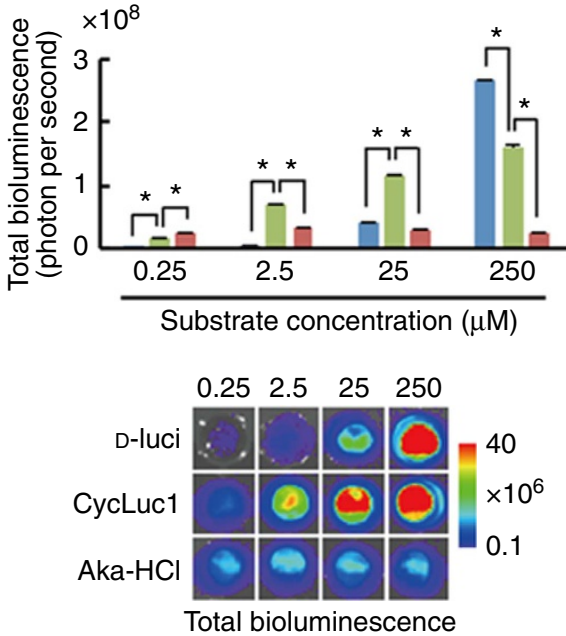

C
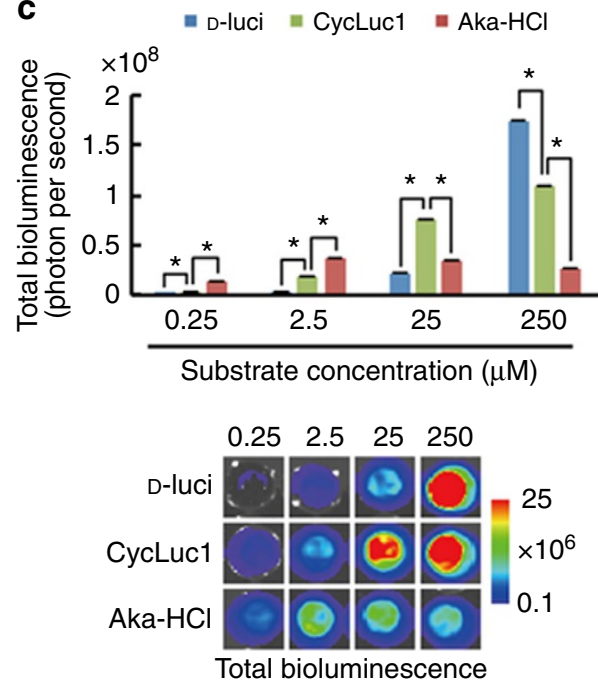

b

= D-luci $=$ CycLuc1 $=$ Aka-HCl
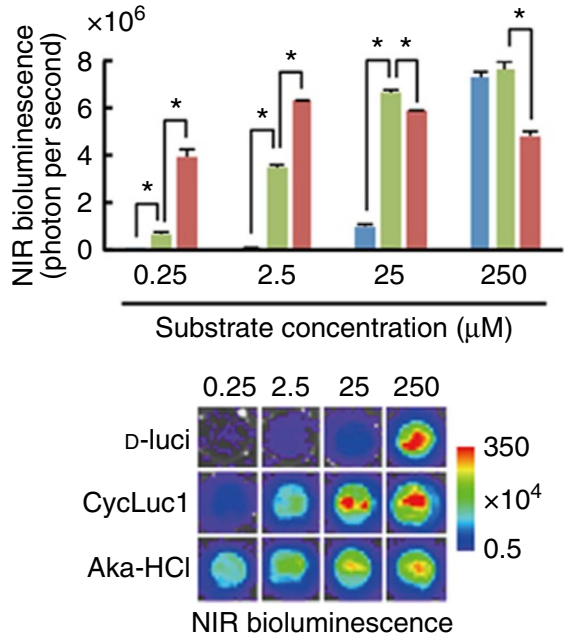

d
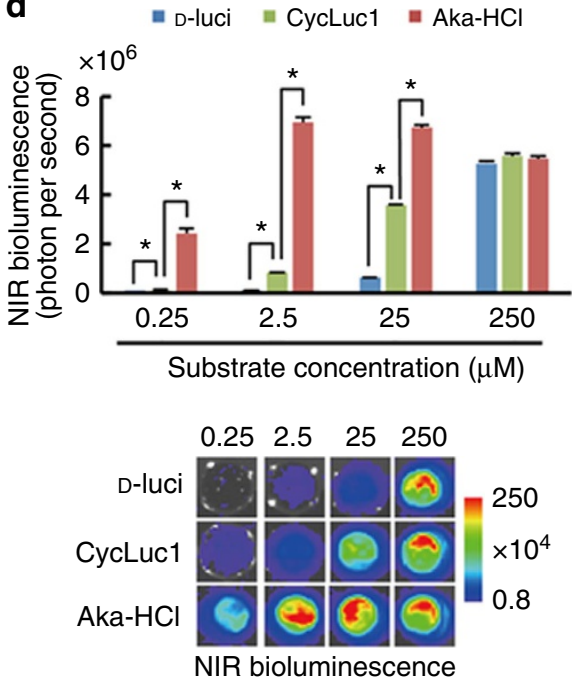

Figure 2 | Cellular imaging using AkaLumine-HCl. Substrate dose dependency of total (a,c) or NIR (b,d) bioluminescence production in LLC/luc $(\mathbf{a}, \mathbf{b})$ or MDA-MB-231/luc $(\mathbf{c}, \mathbf{d})$ cells. The cells $\left(4 \times 10^{5}\right.$ cells per well) were treated with the substrate at indicated concentrations. Images were acquired with $680 \pm 10 \mathrm{~nm}$ emission filter (right panels) or without a filter (left panels), to measure NIR bioluminescence or total bioluminescence signals, respectively. $n=3,{ }^{\star} P<0.05$ (t-test). Error bars indicate s.e.m. 
Table 1 | Parameters of enzymatic reaction of luciferase substrate.

\begin{tabular}{lcc} 
& $\boldsymbol{K}_{\mathbf{m}} \pm$ s.e.m. $(\boldsymbol{\mu M})$ & $\boldsymbol{V}_{\mathbf{m a x}} \pm$ s.e.m. $\left(\times \mathbf{1 0}^{\mathbf{6}}\right)$ \\
\hline D-luci & $107 \pm 14.7$ & $13.8 \pm 0.71$ \\
CycLuc1 & $1.06 \pm 0.06$ & $7.00 \pm 0.09$ \\
Aka-HCl & $2.06 \pm 0.63$ & $2.07 \pm 0.23$ \\
\hline
\end{tabular}

Aka- $\mathrm{HCl}$, AkaLumine- $\mathrm{HCl}, \mathrm{CycLuc1,} \mathrm{cyclic} \mathrm{alkylaminoluciferin;} \mathrm{D-luci,} \mathrm{D-luciferin.}$

$K_{m}$ and $V_{\max }$ values of D-luci, CycLucl and Aka-HCl. These values were calculated from reactions with recombinant Fluc protein and the substrate in the presence of Mg-ATP.

a

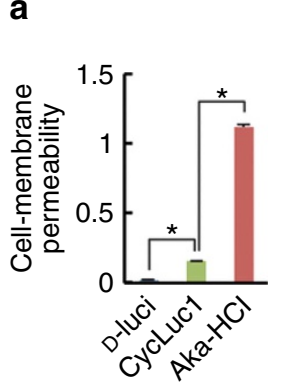

b

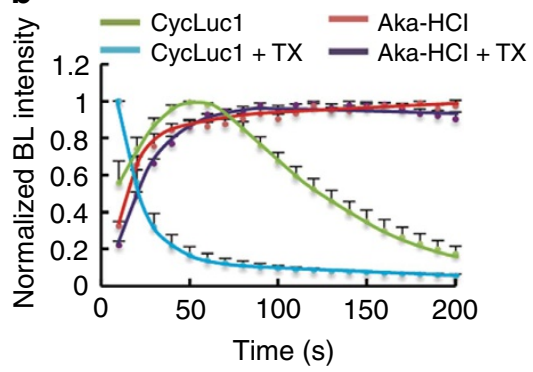

C

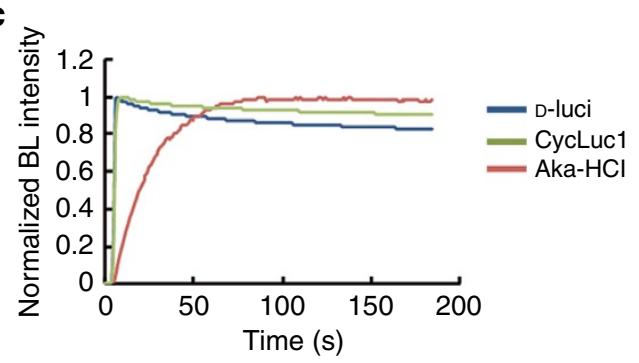

Figure 3 | Cell-membrane permeability of AkaLumine-HCl. (a) Cellmembrane permeability of the substrates. Ratio of bioluminescence (BL) intensity indicates relative bioluminescence intensity of intact cells versus lysed cells treated with indicated substrates at $2.5 \mu \mathrm{M} . n=3,{ }^{\star} P<0.05$ ( $t$-test). (b) BL production in LLC/luc cells with or without pretreatment with $0.1 \%$ Triton X-100 (TX). BL intensity was measured every $10 \mathrm{~s}$ after addition of $\mathrm{CycLuc1}$ or AkaLumine- $\mathrm{HCl}(\mathrm{Aka}-\mathrm{HCl})$ (final concentration of $25 \mu \mathrm{M}$ ). BL intensity at each time point was normalized to the peak intensity. $n=3$. (c) BL production with recombinant Fluc protein. The substrates (final concentration of $25 \mu \mathrm{M}$ ) were reacted with recombinant Fluc proteins $\left(20 \mu \mathrm{g} \mathrm{ml}^{-1}\right)$. The reaction was initiated by the addition of ATP-Mg (final concentration of $80 \mu \mathrm{M}$ ). Data are representative of three independent experiments. Error bars indicate s.e.m.

Triton-X-100 greatly accelerated the bioluminescence production rate of CycLuc1 (Fig. 3b), revealing that the membrane permeability is the rate-limiting factor of the bioluminescence production by CycLucl. On the other hand, AkaLumine- $\mathrm{HCl}$ showed similar bioluminescence production kinetics in intact or detergent-treated cells and in the reaction with recombinant Fluc protein (Fig. 3b,c). Taken together, small $K_{\mathrm{m}}$ value and high cellmembrane permeability may be key factors for distinct bioluminescence production by AkaLumine- $\mathrm{HCl}$ in cells. This property is a significant advantage over D-luciferin and CycLucl for bioluminescence animal imaging, as variation in substrate bioavailability among tissues is one of the main issues hindering the acquisition of quantitative information of targets located in different parts of the body. In addition, we also confirmed the linear correlation of signal output with increasing numbers of LLC/luc and MDA-MB-231/luc cells following treatment with $25 \mu \mathrm{M}$ AkaLumine-HCl (Supplementary Fig. 4).
Highly sensitive in vivo BLI using AkaLumine-HCl. We then sought to assess the suitability of AkaLumine- $\mathrm{HCl}$ for in vivo BLI. AkaLumine-HCl emitted NIR-shifted bioluminescence from subcutaneous tumours with a similar spectrum to that obtained in the reactions with recombinant Fluc protein (Fig. 4a). In a time course of bioluminescence production in vivo, AkaLumine- $\mathrm{HCl}$ displayed a stable glow-type reaction at various injection doses and its bioluminescence production remained elevated longer at the higher concentrations (Supplementary Fig. 5); this temporally more stable bioluminescence production may be beneficial for acquiring images without influence of acquisition time. Furthermore, AkaLumine- $\mathrm{HCl}$ has a relatively longer half-life ( $\sim 40 \mathrm{~min}$ ) in serum (Fig. $4 \mathrm{~b})$. These results suggest that AkaLumine-HCl would be a suitable substrate for in vivo imaging. To address this issue, we first compared the differences in in vivo detection sensitivity between bioluminescence signals generated by $\mathrm{D}$-luciferin and AkaLumine- $\mathrm{HCl}$ in a subcutaneous tumour, which is one of the most easily detectable surface targets, after intraperitoneal injection with various doses of these substrates. AkaLumine- $\mathrm{HCl}$ was injected into the mice $4 \mathrm{~h}$ after D-luciferin injection, when D-luciferin bioluminescence was negligible (Supplementary Fig. 6a). The bioluminescence signals produced by AkaLumine-HCl were $>40$-fold higher than those of D-luciferin after injecting $1 \mathrm{mM}$ substrates (Fig. 4c,d). This improvement in the detection of subcutaneous tumours is comparable to CycLucl (ref. 9). We then compared bioluminescence production between AkaLumine- $\mathrm{HCl}$ and CycLucl in subcutaneous tumours by intraperitoneal injection of $5 \mathrm{mM}$ substrate into the same mice in order of CycLucl and AkaLumine- $\mathrm{HCl}$ at an $8-\mathrm{h}$ interval, which was long enough to make CycLuc1 bioluminescence negligible (Supplementary Fig. 6b). Detection sensitivity of surface targets was comparable between $5 \mathrm{mM}$ AkaLumine-HCl and CycLucl (Supplementary Fig. 7), reflecting the fact that the signals from the body surface are less influenced by tissue penetration efficiency and thus receive less benefit from NIR bioluminescence.

Improved deep tissue imaging using AkaLumine-HCl. We then address whether AkaLumine- $\mathrm{HCl}$ could improve the detection sensitivity for targets located in deep tissues such as metastasis in mouse lung, which is one of the most difficult tissues for optical imaging due to high scattering and absorption of light ${ }^{18}$, as well as its location deep within the body (Fig. 5a,b). Bioluminescence images were acquired from the same mice bearing LLC/luc lung metastases after intraperitoneal injection in order of $33 \mathrm{mM}$ D-luciferin and AkaLumine- $\mathrm{HCl}$ at a 4-h interval. Notably, AkaLumine-HCl displayed greatly enhanced signals emanating from metastasis in the lung (Fig. 5c), as demonstrated by 8.1 -fold higher with AkaLumine- $\mathrm{HCl}$ as compared with D-luciferin administration (Fig. 5c). To evaluate the advantage of AkaLumine- $\mathrm{HCl}$ over CycLuc1 in detecting targets in deep tissue, we then compared bioluminescence images between AkaLumine-HCl- and CycLucl-treated mice $15 \mathrm{~min}$ after intravenous injection of LLC/luc cells at the dose of $5 \mathrm{mM}$, 
a

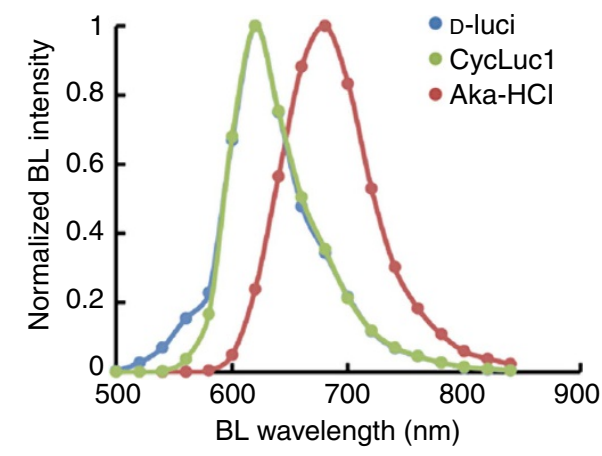

C

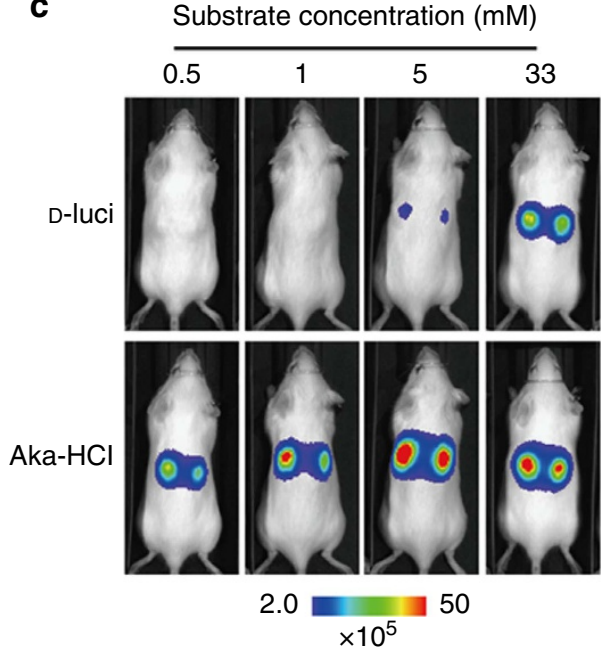

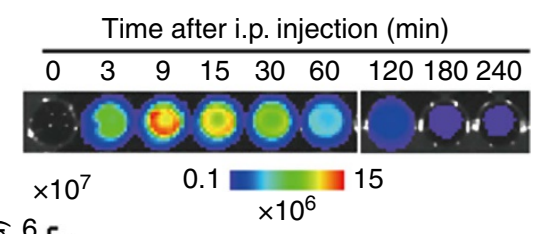

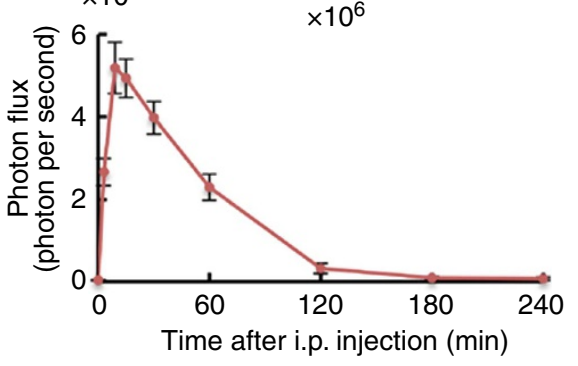

d

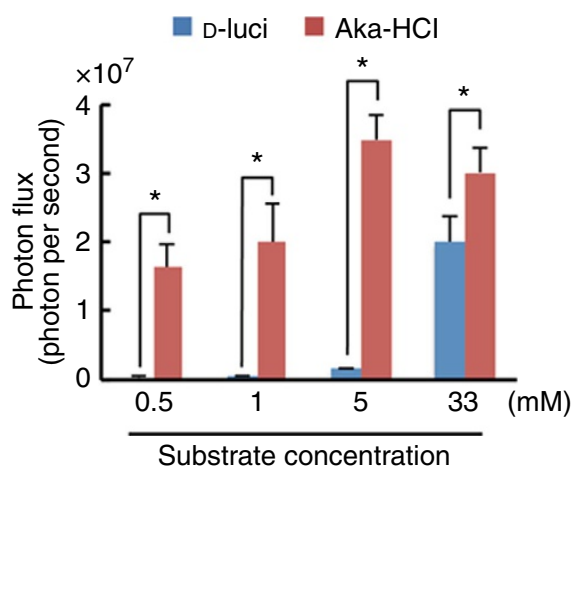

Figure 4 | In vivo BLI of cancer cells using AkaLumine-HCl. (a) Bioluminescence spectrum from subcutaneous tumours. Bioluminescence (BL) intensity was measured with 18 filters $(500-840 \mathrm{~nm}$ ) in IVIS Spectrum after injection of D-luciferin (D-luci), CycLuc1 and AkaLumine- $\mathrm{HCl}(\mathrm{Aka}-\mathrm{HCl})$ into mice bearing subcutaneous LLC/luc tumours. Date are representative of three independent experiments. (b) Half-life of AkaLumine-HCl in serum. Recombinant Fluc proteins were reacted with blood sampled at indicated time after intraperitoneal injection of $5 \mathrm{mM} \mathrm{AkaLumine-HCl}$. Representative $\mathrm{BL}$ images (upper) and quantitative analysis (bottom) of BL intensity generated by reaction with recombinant Fluc proteins and AkaLumine- $\mathrm{HCl}$ in sampled blood are shown. $n=3$. (c) Representative BL images of LLC/luc subcutaneous tumours and (d) quantitative analysis of BL production 15 min after intraperitoneal injection of $100 \mu$ l of D-luciferin (D-luci) or AkaLumine- $\mathrm{HCl}\left(\mathrm{Aka}-\mathrm{HCl}\right.$ ) with indicated concentration. $n=6,{ }^{\star} P<0.05$ (t-test). The substrates were injected to the same mouse in the order of $\mathrm{D}-\mathrm{luci}$ and $\mathrm{Aka}-\mathrm{HCl}$ at a 4-h interval. Error bars indicate s.e.m.

which is the maximum concentration of CycLuc1 due to its low water solubility (Fig. 5d). AkaLumine-HCl exhibited 3.3-fold increase in detection sensitivity of disseminated cancer cells in the lung compared with CycLuc1 (Fig. 5d). The advantage of AkaLumine- $\mathrm{HCl}$ was further confirmed by imaging the same mice bearing lung metastasis after intraperitoneal injection of $5 \mathrm{mM}$ substrates in order of CycLucl and AkaLumine- $\mathrm{HCl}$ (Fig. 5e), and in the inverse order (Supplementary Fig. 8) at an 8-h interval. AkaLumine- $\mathrm{HCl}$ displayed about fourfold increase in signals from lung metastasis compared with CycLuc1 (Fig. 5e and Supplementary Fig. 8).

\section{Discussion}

In this study, we demonstrate that the bioluminescence produced by AkaLumine- $\mathrm{HCl}$ achieved considerable penetration depths (Figs $1 \mathrm{c}$ and $5 \mathrm{c}-\mathrm{e}$ ) and exhibited small temporal fluctuation (Supplementary Fig. 5), much less influenced by its concentration (Figs 2 and $4 \mathrm{~d}$ ). The properties that enable more sensitive detection of targets in deep tissues and more accurate quantitative comparison of targets localizing to different parts of the body are significant advantages of AkaLumine- $\mathrm{HCl}$ over D-luciferin and CycLucl, and therefore AkaLumine- $\mathrm{HCl}$ might become the preferred BLI substrate in preclinical studies. The small temporal fluctuation in signal production by AkaLumine- $\mathrm{HCl}$ in a mouse model (Supplementary Fig. 5) may be achieved by its properties of lower saturation concentration (Fig. 2), high membrane permeability (Fig. 3) and relatively longer circulation half-time in the serum (Fig. 4b). These properties may contribute to having the reaction rate remain constant in tumour cells for relatively long periods of time, leading to more accurate and efficient quantitative analysis of targets from many mice in limited time.

In this study, we demonstrated that AkaLumine- $\mathrm{HCl}$ significantly improved target detection sensitivity in deep lung metastases (Fig. $5 \mathrm{c}-\mathrm{e}$ ) by overcoming the fundamental limitation of short emission wavelength from D-luciferin and CycLuc1. Of note, a recent study detailed the synthesis of infra-luciferin $\left(\mathrm{iLH}_{2}\right)$, which emits NIR bioluminescence of the longest-known wavelength via its reaction with mutant Fluc ${ }^{10}$. However, the $\mathrm{iLH}_{2}$ signal output was exceedingly low when compared with that of D-luciferin and, therefore, $\mathrm{iLH}_{2}$ requires chemical optimization for practical use in biological studies. AkaLumine- $\mathrm{HCl}$ exhibits a 
a

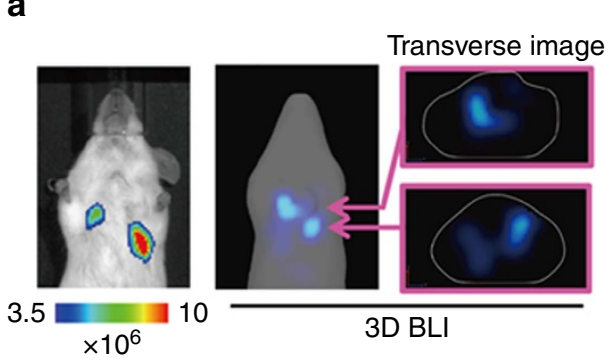

b

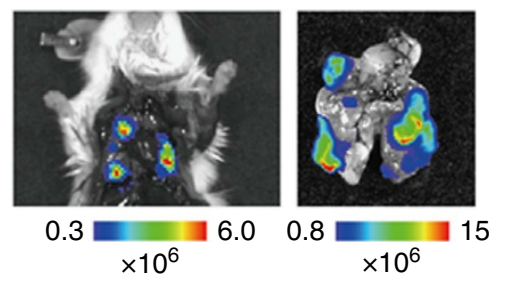

C
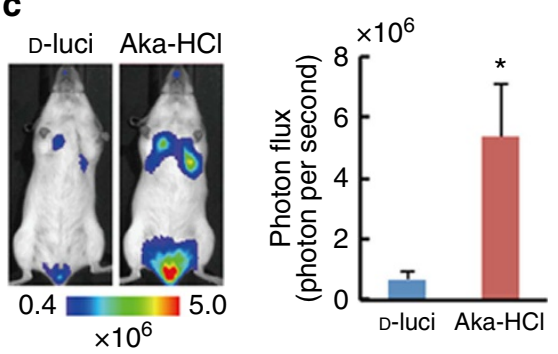

d
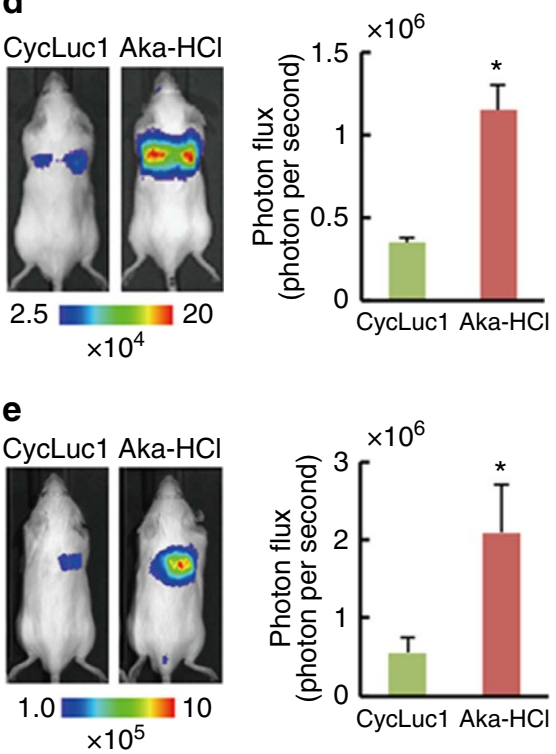

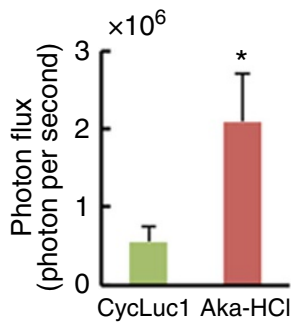

Figure 5 | Highly sensitive in vivo BLI of deep tissue tumours. (a) Typical bioluminescence (BL) images of lung metastasis developed after intravenous injection of LLC/luc cells. Three-dimensional BLI (right panels) showed metastasis developed in deep lung tissues. The images were acquired after intraperitoneal injection of AkaLumine- $\mathrm{HCl}(33 \mathrm{mM})$. (b) Ex vivo BLI of metastatic lesions in the lung after removing sternum (left panel) and removed lung (right panel) of the mouse shown in a. (c) Representative BL images of LLC/luc lung metastasis (left panel) and quantitative analysis of BL production (right panel) at peak time after intraperitoneal injection of $100 \mu \mathrm{l}$ of $33 \mathrm{mM}$ substrates. $n=7,{ }^{\star} P<0.05$ (t-test). (d) Representative BL images of $\mathrm{LLC} /$ luc disseminated in the lung (left panel) and quantitative analysis of $\mathrm{BL}$ production (right panel) at peak time after intraperitoneal injection of $100 \mu \mathrm{l}$ of $5 \mathrm{mM}$ substrates. $n=8,{ }^{\star} P<0.05$ (t-test). Error bars indicate s.e.m. (e) Representative BL images of lung metastasis of LLC/luc (left panel) and quantitative analysis of BL production (right panel) at peak time after intraperitoneal injection of $100 \mu \mathrm{l}$ of $5 \mathrm{mM}$ substrates. $n=8,{ }^{\star} P<0.05$ ( $t$-test). Error bars indicate s.e.m.

considerably high NIR bioluminescence production with native Fluc that is currently used in general in vivo BLI applications. As such, AkaLumine- $\mathrm{HCl}$ would be immediately available for use in a broad range of biological studies that use BLI in small animal models.

\section{Methods}

Synthesis of AkaLumine-HCl. AkaLumine was synthesized as previously described $^{12}$. To prepare AkaLumine- $\mathrm{HCl}, 4 \mathrm{M} \mathrm{HCl}$ in Dioxane $(0.5 \mathrm{ml})$ was added to a suspending solution of the AkaLumine $(20 \mathrm{mg}, 0.066 \mathrm{mmol})$. This mixture solution was centrifuged after vortex for 10 min. The supernatant and the precipitate were then subjected to solid-liquid separation. The obtained precipitate was washed with $1 \mathrm{ml}$ ethylacetate three times and dried in vacuum to obtain the AkaLumine- $\mathrm{HCl}(20 \mathrm{mg}, 0.059 \mathrm{mmol})$ as a light purple solid; $85 \%$ ee from chiral HPLC (retention time of L-isomer: $16.8 \mathrm{~min}$, D-isomer: $17.6 \mathrm{~min}$ ); ${ }^{1} \mathrm{H}$ NMR $\left(500 \mathrm{MHz}, \mathrm{DMSO}-d_{6}\right): d 7.67(d d, J=14.9,11.5 \mathrm{~Hz}, 1 \mathrm{H}), 7.59(d, J=9.16 \mathrm{~Hz}, 2 \mathrm{H})$, $7.38(d, J=15.1 \mathrm{~Hz}, 1 \mathrm{H}), 7.19(d d, J=14.9,11.5 \mathrm{~Hz}, 1 \mathrm{H}), 6.92(\mathrm{br}, 2 \mathrm{H}), 6.81$ $(d, J=14.9 \mathrm{~Hz}, 1 \mathrm{H}), 5.47(d d, J=10.3,5.5 \mathrm{~Hz}, 1 \mathrm{H}), 3.98(d d, J=11.5,5.5 \mathrm{~Hz}, 1 \mathrm{H})$, $3.87(d d, J=11.5,10.3 \mathrm{~Hz}, 1 \mathrm{H}), 3.04(s, 6 \mathrm{H}) ;{ }^{13} \mathrm{C}$ NMR $\left(125 \mathrm{MHz}, \mathrm{DMSO}-d_{6}\right)$ $d$ 182.8, 169.8, 154.3, 150.9, 148.6, 131.0, 126.1, 123.6, 114.5, 66.5, 41.5, 34.39.

The optical purity of synthesized luciferin analogues was analysed by Thermo LC-MS (LCQ Fleet) using a chiral column (Daicel Chemical Industries, OZ-RH, $5 \mathrm{~mm}, 4.6-150 \mathrm{~mm}$ ) with a linear gradient of $10-90 \%$ acetonitrile in $0.05 \%$ TFA in $\mathrm{H}_{2} \mathrm{O}$ over $30 \mathrm{~min}$ (flow rate $0.5 \mathrm{ml} \mathrm{min}{ }^{-1}$ ) as eluent. ${ }^{1} \mathrm{H}$ and ${ }^{13} \mathrm{C}$ NMR spectra were recorded on JEOL ECA 500 instruments. Chemical shifts are reported in parts per million $(d)$ downfield from internal tetramethylsilane $\left(d \frac{1}{4} 0\right)$ and coupling constants in Hertz. Infrared spectra were measured by Nicolet 6700 FT-IR Spectrometer using attenuated total reflection methods. AkaLumine- $\mathrm{HCl}$ (trade name: TokeOni, 808350-5MG) can be obtained from Sigma-Aldrich (St Louis, MO, USA).

Measurement of bioluminescence emission spectra. Bioluminescence emission spectra of D-luciferin (Promega, Madison, WI, USA), CycLucl (AOBIOUS INC., Gloucester, MA, USA) and AkaLumine- $\mathrm{HCl}$ were measured using an ATTO AB-1850 spectrophotometer (ATTO Co. Ltd, Tokyo, Japan). A reaction mixture was prepared by mixing $5 \mu \mathrm{l}$ of a substrate $(100 \mu \mathrm{M}), 5 \mu \mathrm{l}$ of QuantiLum Recombinant Luciferase solution $\left(1 \mathrm{mg} \mathrm{ml}^{-1}\right.$ ) (Promega) and $5 \mu \mathrm{l}$ of potassium phosphate buffer $(500 \mathrm{mM}, \mathrm{pH} 8.0)$. Luminescence reactions were then initiated by injecting $10 \mu \mathrm{l}$ of ATP-Mg $(200 \mu \mathrm{M})$ into the reaction mixture. Bioluminescence emission spectra were measured in $1 \mathrm{~nm}$ increments from 400 to $780 \mathrm{~nm}$ using $3 \mathrm{~min}$ of integration time.

Measurement of $\boldsymbol{K}_{\mathbf{m}}$ value. The bioluminescence intensities of D-luciferin, CycLucl and AkaLumine- $\mathrm{HCl}$ were measured in same conditions with measurement of bioluminescence spectrum, except substrate concentration. Final concentrations of the substrates were varied from 0.2 to $500 \mu \mathrm{M}$ and 0.1 to $100 \mu \mathrm{M}$, respectively. $K_{\mathrm{m}}$ and $V_{\max }$ values of the substrates were determined from the integrated value of the bioluminescence intensity and calculated by the Lineweaver-Burk plots by using the Enzyme Kinetics Wizard in the commercially available SigmaPlot 9.0 software package (Systat Software Inc., San Jose, CA, USA). 
Bioluminescence transmission assay using biological tissues. Bioluminescence signal from wells was measured with IVIS Spectrum (PerkinElmer, Boston, MA USA) $1 \mathrm{~min}$ after the substrate (final concentration of $2.5 \mu \mathrm{M}$ for D-lucfierin and AkaLumine-HCl, $50 \mathrm{nM}$ for CycLuc1) was reacted with recombinant Fluc proteins $\left(20 \mu \mathrm{g} \mathrm{ml}^{-1}\right)$ in the presence of ATP-Mg (final concentration of $20 \mu \mathrm{M}$ ) in a black 96-well plate. A biological tissue (4-mm-thick sliced beef) was placed on the wells, to measure bioluminescence signal through the tissue, followed by acquiring bioluminescence images through two layers of the biological tissue. The following conditions were used for image acquisition: exposure time $=10 \mathrm{~s}$, binning $=$ medium: 8 , field of view $=12.9 \times 12.9 \mathrm{~cm}$, and $f / \mathrm{stop}=1$. The bioluminescence images were analysed by Living Image 4.3 software (PerkinElmer) specialized for IVIS system. Penetration efficiency (\%) was calculated by dividing the signal intensities of the beef-covered wells by those of the corresponding uncovered wells.

Isolation of cancer cell lines stably expressing luc reporters. The murine lung carcinoma cell LLC, human breast cancer cell MDA-MB-231 and human prostate cancer cell PC-3 were obtained from ATCC (Rockville, MD, USA). LLC/luc and MDA-MB-231/luc cells were isolated after transfection with plasmid $\mathrm{pEF} / \mathrm{luc}$ by calcium phosphate method as previously described ${ }^{19,20}$. PC-3/ $\mathrm{\kappa B}$-luc was also isolated as previously described ${ }^{21}$. The cells were maintained at $37^{\circ} \mathrm{C}$ in $5 \%$ FCS-DMEM (Nacalai Tesque, Kyoto, Japan) supplemented with penicillin (100 units per $\mathrm{ml}$ ) and streptomycin $\left(100 \mu \mathrm{g} \mathrm{ml}^{-1}\right)$ and regularly checked for mycoplasma contamination by a mycoplasma check kit (Lonza, Basel,

Switzerland). All the cell lines were independently stored and recovered from the original stock every time for each experiment.

In vitro BLI. The substrates were reacted with LLC/luc or MDA-MB-231/luc cells $\left(4 \times 10^{5}\right.$ cells per well) in a black 96-well plate. Bioluminescence was measured using IVIS Spectrum $1 \mathrm{~min}$ after adding the substrates. The following conditions were used for image acquisition: open for total bioluminescence or $680 \pm 10 \mathrm{~nm}$ of an emission filter for NIR bioluminescence, exposure time $=10 \mathrm{~s}$, binning $=$ medium: 8 , field of view $=12.9 \times 12.9 \mathrm{~cm}$ and $f / \mathrm{stop}=1$. The bioluminescence images were analysed by Living Image 4.3 software (PerkinElmer) specialized for IVIS system.

Cell-membrane permeability assay. Bioluminescence from lysed or intact LLC/luc ( $10^{5}$ cells) was measured in a black 96-well plate using IVIS Spectrum. Lysed cells were prepared with $2 \times$ Passive Lysis Buffer (Promega) supplemented with Tris- $\mathrm{HCl}(20 \mathrm{mM})$, EDTA $(0.2 \mathrm{mM})$, ATP-Mg $(500 \mathrm{nM})$, BSA $\left(6 \mathrm{mg} \mathrm{ml}^{-1}\right)$ and dithiothreitol $(33 \mathrm{mM})$. The measurement was performed $1 \mathrm{~min}$ after adding the substrates (final concentration of $2.5 \mu \mathrm{M}$ ). The following conditions were used for image acquisition: open emission filter, exposure time $=10 \mathrm{~s}$, binning $=$ medium: 8 , field of view $=12.9 \times 12.9 \mathrm{~cm}$ and $f / \mathrm{stop}=1$. The bioluminescence images were analysed by Living Image 4.3 software (PerkinElmer) specialized for IVIS system. Bioluminescence emission from LLC/luc $\left(5 \times 10^{5}\right.$ cells) with or without pretreatment of $0.1 \%$ Triton-X-100 for $3 \mathrm{~min}$ was measured every $10 \mathrm{~s}$ for $200 \mathrm{~s}$ using a luminometer GL-210A (Microtec Co., Ltd, Chiba, Japan). Bioluminescence measurement was started by injection of the substrate (final concentration of $25 \mu \mathrm{M})$.

Mice. C57B/6 albino mice (female and male) or severe combined immunodeficient mice (male) were obtained from Charles River Laboratory Japan (Yokohama, Japan). All mice used were littermates or age-matched (7 weeks of age) females or males, were provided access to food and water ad libitum and were housed in the animal facilities at Tokyo Institute of Technology.

All the experimental procedures using mice were approved by the Animal Experiment Committees of Tokyo Institute of Technology (authorization numbers 2010006-3 and 2014005) and carried out in accordance with relevant national and international guidelines.

Tumour models. For subcutaneous tumour models, LLC/luc $\left(3 \times 10^{5}\right.$ cells per $10 \mu \mathrm{l})$ or PC-3/KB-luc $\left(1 \times 10^{6}\right.$ cells per $\left.10 \mu \mathrm{l}\right)$ suspended in PBS was mixed with an equal volume of Geltrex (Invitrogen) and subcutaneously or intratibially injected into C57B/6 albino mice (female) or severe combined immunodeficient mice (male), respectively. The experiments were performed 4 days after engraftment. For a model with disseminated cancer cells in the lung, C57B/6 albino mice (male) were intravenously injected with LLC/luc $\left(1 \times 10^{5}\right.$ cells per $100 \mu \mathrm{l}$ PBS $) 15$ min before in vivo BLI. For lung metastasis model, LLC/luc $\left(5 \times 10^{5}\right.$ cells per $\left.100 \mu \mathrm{l}\right)$ suspended in PBS was injected from tail vein of C57B/6 albino mice (male). The experiments were performed 10-20 days after intravenous injection. These tumour models are well established and tumour growth is stable. Therefore, six samples are adequate sample size for evaluation of tumour growth in each experiment.

In vivo BLI. Bioluminescence images of subcutaneous tumours were acquired with IVIS Spectrum $15 \mathrm{~min}$ (unless otherwise indicated) after intraperitoneal injection with indicated amount of the substrates. As bioluminescence intensities from lung metastasis peaked at various time after a substrate injection, bioluminescence images of lung metastasis were sequentially acquired with IVIS Spectrum every $3 \mathrm{~min}$ for $30 \mathrm{~min}$ after intraperitoneal injection with the substrates and the highest bioluminescence intensities among the acquired images were selected for analysis. For comparing bioluminescence production between different substrates using the same mice, the images for AkaLumine- $\mathrm{HCl}$ were acquired 4 and $8 \mathrm{~h}$ after injection of D-luciferin and CycLucl, respectively. The following conditions were used for image acquisition: open emission filter, exposure time $=60 \mathrm{~s}$, binning $=$ medium: 8 , field of view $=12.9 \times 12.9 \mathrm{~cm}$ and $f /$ stop $=1$. For three-dimensional BLI in lung metastasis model, a mouse injected with AkaLumine- $\mathrm{HCl}$ was subjected to BLI with three different wavelengths $(660 \pm 10,680 \pm 10$ and $700 \pm 10 \mathrm{~nm})$ of bioluminescence filters. The following conditions were used for imaging acquisition: exposure time $=60 \mathrm{~s}$, binning $=$ medium: 8 , field of view $=12.9$ $\times 12.9 \mathrm{~cm}$ and $f /$ stop $=1$. The bioluminescence images were analysed by Living Image 4.3 software (PerkinElmer) specialized for IVIS system.

Ex vivo BLI. A mouse was scarified immediately after in vivo BLI by using AkaLumine- $\mathrm{HCl}$ and the lung was removed. Bioluminescence image of the lung was obtained with the following conditions: open emission filter, exposure time $=30 \mathrm{~s}$, binning $=$ medium: 8 , field of view $=12.9 \times 12.9 \mathrm{~cm}$ and $f / \mathrm{stop}=1$. The bioluminescence images were analysed by Living Image 4.3 software (PerkinElmer) specialized for IVIS system.

Measurement of AkaLumine-HCl half-life in serum. Five microlitres of blood was sampled from tail vein and then mixed with $45 \mu \mathrm{l}$ PBS, $25 \mu \mathrm{l}$ recombinant Fluc protein $\left(20 \mu \mathrm{g} \mathrm{ml}^{-1}\right)$ and $25 \mu \mathrm{l}$ ATP-Mg $(80 \mu \mathrm{M})$ in a black 96-well plate, to measure bioluminescence intensity by IVIS Spectrum. The following conditions were used for image acquisition: exposure time $=10 \mathrm{~s}$, binning $=$ medium, field of view $=12.9 \times 12.9$ and $f /$ stop $=1$. The bioluminescence images were analysed by Living Image 4.3 software.

Statistical analysis. Data are presented as means \pm s.e.m. and were statistically analysed with a two-sided Student's $t$-test. $P$-values $<0.05$ were considered statistically significant.

Data availability. The data that support the findings of this study are available from the corresponding author on request.

\section{References}

1. Fraga, H. Firefly luminescence: a historical perspective and recent developments. Photochem. Photochem. Photobiol. Sci. 7, 146-158 (2008).

2. Inouye, S. Firefly luciferase: an adenylate-forming enzyme for multicatalytic functions. Cell. Mol. Life Sci. 67, 387-404 (2010).

3. Rehemtulla, A. et al. Rapid and quantitative assessment of cancer treatment response using in vivo bioluminescence imaging. Neoplasia 2, 491-495 (2000).

4. Luker, K. E. et al. Kinetics of regulated protein-protein interactions revealed with firefly luciferase complementation imaging in cells and living animals. Proc. Natl Acad. Sci. USA 101, 12288-12293 (2004).

5. Harada, H., Kizaka-kondoh, S. \& Hiraoka, M. Optical imaging of tumor hypoxia and evaluation of efficacy of a hypoxia-targeting drug in living animals. Mol. Imaging Biol. 4, 182-193 (2005).

6. Ozaki, M., Haga, S. \& Ozawa, T. In vivo monitoring of liver damage using caspase-3 probe. Theranostics 2, 207-214 (2012).

7. Weissleder, R. \& Ntziachristos, V. Shedding light onto live molecular targets. Nat. Med. 9, 123-128 (2003)

8. Dawson, B. et al. A theoretical and experimental study of light absorption and scattering by in vivo skin. Phys. Med. Biol. 25, 695-709 (1980).

9. Evans, M. S. et al. A synthetic luciferin improves bioluminescence imaging in live mice. Nat. Methods 11, 393-395 (2014).

10. Jathoul, A. P., Grounds, H., Anderson, J. C. \& Pule, M. A. A dual-color far-red to near-infrared firefly luciferin analogue designed for multiparametric bioluminescence imaging. Angew. Chem. Int. Ed. Engl. 126, 1-6 (2014),

11. Kojima, R. et al. Rational design and development of near-infrared-emitting firefly luciferins available in vivo. Angew. Chem. Int. Ed. Engl. 52, 1175-1179 (2013).

12. Iwano, S. et al. Development of simple firefly luciferin analogs emitting blue, green, red, and near-infrared biological window light. Tetrahedron $\mathbf{6 9}$, 3847-3856 (2013).

13. Fontes, R., Dukhovich, A., Sillero, A. \& Sillero, M. A. Synthesis of dehyroluciferin by firefly luciferase: effect of dehyroluciferin, coenzyme A and nucleoside riphosphates on the luminescent reaction. Biochem. Biophys. Res. Commun. 237, 445-450 (1997).

14. Ribeiro, C. \& Esteves da Silva, J. C. Kinetics of inhibiton of firefly luciferase by oxyluciferin and dehyroluciferyl-adenylate. Photochem. Photobiol. Sci. 7, 1085-1090 (2008).

15. Craig, F. F., Simmonds, A. C., Watmore, D., Mccaprat, F. \& White, M. R. H. Membrane-permable luciferin esters for assay of firefly luciferase in live intact cells. Biochem. J. 276, 637-641 (1991). 
16. Shinde, R., Perkins, J. \& Contag, C. H. Luciferin derivatives for enhanced in vitro and in vivo bioluminescence assays. Biochemistry 45, 11103-11112 (2006).

17. Mofford, D. M., Reddy, G. R. \& Miller, S. C. Aminoluciferins extend firefly luciferase bioluminescence into near-infrared and can be prefrerred substrates over D-luciferin. J. Am. Chem. Soc. 136, 13277-13282 (2014).

18. Cheong, W. F., Prahl, S. A. \& Welch, A. J. A review of optical properties of biological tissues. IEEE J. Quantum Electron. 26, 2166-2185 (1980).

19. Chen, C. \& Okayama, H. High-efficiency transformation of mammalian cells by plasmid DNA. Mol. Cell. Biol. 7, 2745-2752 (1987).

20. Kizaka-Kondoh, S. et al. Selective killing of hypoxia-inducible factor-1-active cells improves survival in a mouse model of invasive and metastatic pancreatic cancer. Clin. Cancer Res. 15, 3433-3441 (2009).

21. Fujita, K. et al. Intracelluar CO release from composite of ferritin and ruthenium carbonyl complexes. J. Am. Chem. Soc. 136, 16902-16908 (2014).

\section{Acknowledgements}

We are grateful to Shigeaki Watanabe and Machiko Horiuchi (Summit Pharmaceuticals International Corporation, Tokyo, Japan) for discussion and technical support of IVIS. We are also grateful to Kuroganekasei Co. Ltd for synthesis of AkaLumine. This research was supported by a Grant-in-Aid for Scientific Research on Innovative Areas 'Integrative Research on Cancer Microenvironment Network' from the Ministry of Education, Culture, Sports, Science and Technology of Japan (S.K.-K.), Adaptable and Seamless Technology Transfer Program through target-driven R\&D JST and a Grant-in-Aid for challenging Exploratory Research (S.Ma).

\section{Author contributions}

T.Ku. and S.I. contributed equally to this work. S.K.-K., S.Ma and H.N. designed and managed the overall project. T.Ku, S.M. and T.Ka. performed imaging experiments using cultured cells and animals. S.I. and M.K. synthesized AkaLumine-HCl and characterized the substrates in a test tube. T.Ku, S.I., H.N., S.Ma and S.K.-K. wrote the manuscript.

\section{Additional information}

Supplementary Information accompanies this paper at http://www.nature.com/ naturecommunications

Competing financial interests: The authors declare no competing financial interests.

Reprints and permission information is available online at http://npg.nature.com/ reprintsandpermissions/

How to cite this article: Kuchimaru, T. et al. A luciferin analogue generating near-infrared bioluminescence achieves highly sensitive deep-tissue imaging. Nat. Commun. 7:11856 doi: 10.1038/ncomms11856 (2016).

\section{(c) (1)}

This work is licensed under a Creative Commons Attribution 4.0 International License. The images or other third party material in this article are included in the article's Creative Commons license, unless indicated otherwise in the credit line; if the material is not included under the Creative Commons license, users will need to obtain permission from the license holder to reproduce the material. To view a copy of this license, visit http://creativecommons.org/licenses/by/4.0/ 